\title{
Foreword
}

\section{Does the definition of the Mediterranean diet need to be updated?}

\author{
Lluís Serra-Majem*, Antonia Trichopoulou, Joy Ngo de la Cruz, Pilar Cervera, \\ Alicia García Álvarez, Carlo La Vecchia, Aicha Lemtouni and Dimitrios Trichopoulos, \\ on behalf of the International Task Force on the Mediterranean Diet
}

The term 'Mediterranean diet' reflects the dietary patterns characteristic of several countries in the Mediterranean Basin during the early 1960s. The association between greater longevity and reduced mortality and morbidity for coronary heart disease, certain cancers and other nutrition-related diseases, and the common dietary food patterns in these countries, have substantiated this concept $t^{1,2}$.

Such patterns were defined in 1993 at the International Conference on the Diets of the Mediterranean, having also been previously defined in other meetings ${ }^{1-4}$. They comprise:

- Abundant plant foods (fruits, vegetables, breads, other forms of cereals, beans, nuts and seeds);

- Minimally processed, seasonally fresh and locally grown foods;

- Fresh fruit as the typical daily dessert, with sweets based on nuts, olive oil and concentrated sugars or honey consumed during feast days;

- Olive oil as the principal source of dietary lipids;

- Dairy products (mainly cheese and yoghurt) consumed in low to moderate amounts;

- Fewer than four eggs consumed per week;

- Red meat consumed in low frequency and amounts;

- Wine consumed in low to moderate amounts, generally with meals.

However, it must be said that this characteristic definition of the Mediterranean diet and its typical composition is not without ambiguities, which require certain consideration $^{5,6}$.

Due to multiple factors such as globalisation and economic development, changes in food consumption have influenced and are still challenging traditional healthy food patterns in Mediterranean countries 5 . Epidemiological studies are essential in order to detect trends and associations between food and health, as well as to provide information for nutrition interventions ${ }^{5}$. For example, the development of food-based dietary guidelines requires consumption data to identify commonly consumed foods and establish nutrient profiles for determining food groups, portion sizes and frequency recommendations ${ }^{6}$.

Since 1960, certain health indicators have worsened in Mediterranean countries whereas others have improved, and in general life expectancy has increased. This has been due to a marked decrease in cerebrovascular disease and some cancers such as that of the stomach - a phenomenon seen in other developed countries, and a decline in ischaemic heart disease, which have reduced overall mortality in spite of increased mortality due to other cancers as well as the rise in HIV/AIDS. During this period, obesity has increased because of decreased daily physical activity and increased energy intake. Taking this context into consideration, it is necessary to reflect on the adequacy of establishing criteria for defining the Mediterranean diet as occurring in the 1960s, instead of doing so for periods before or after this time. For example, in Spain at the beginning of the 1960s, large quantities of cooking lard were utilised and consumed, and fruit intake was half that observed at the end of the twentieth century $^{2,6}$. Additionally, vitamin and mineral supplements and fortified foods are commonly used even among those Mediterranean countries where fresh fruits and vegetables are abundantly available.

The prevailing food model in Mediterranean countries may be the one associated with the highest levels of health. With the objective of exploring definitions of the modern Mediterranean diet, the intent of this workshop was to outline the possibility of defining the Mediterranean diet with a certain openness that would acknowledge healthy changes within this model that may have been produced over the last 40 years, or that may come about in the future.

The questions 'Is the traditional always better than the modern?' and 'What are the synergies and/or antagonisms of the modern Mediterranean diet?' guided the initial discussion of this first meeting of the International Task Force on the Mediterranean Diet of the Foundation for the Advancement of the Mediterranean Diet (www.dietamediterranea.com), which took place on 5 March 2002, at the Scientific Park of the University of Barcelona (www.pcb.ub.es). The Task Force was subsequently divided into two working subgroups whose aims were to reach determined points of consensus.

Objectives for this initial meeting were:

- To define and reach consensus on the qualitative aspects of the Mediterranean diet and to update graphics and contents to current, twenty-first century circumstances. 
- To quantify amounts for the various food groups, establishing upper (or lower) consumption levels in view of contemporary knowledge of the relationships between nutrition and health.

- To establish criteria for identifying a given food product as being a constituent of the Mediterranean diet. This definition would be useful for updating the currently delineated composition of the Mediterranean diet as well as to support initiatives from the food and catering sectors.

The recommendations obtained from the workgroups were as follows.

1. To update foods included in messages (such as food-based dietary guidelines) that reflect the latest scientific evidence. For example, the evidence for wine and certain types of meat, traditionally presented in a less favourable light, warrants reassessment of recommendations for these products. In the latter case, varying processing methods and resultant nutrient composition justifies the need for various categories of meat classification. The predominant position of pasta and breads may need to be reconsidered, and whole grains emphasised. Blue fish is an example of another food whose nutritional qualities warrant additional attention.

2. To incorporate messages as part of the graphic that would not only represent food habits but also those lifestyle factors associated with Mediterranean culture, such as physical activity and relaxed pleasant meals shared in a social context. As such, the goal is to transmit the concept of a Mediterranean 'essence', taking into account the reality that several versions of the Mediterranean diet exist, from which diverse culinary traditions are derived. As such, a constellation of related factors would be acknowledged.

3. To develop qualitative and positive recommendations, emphasising what to consume and not so much what to avoid.

4. To increase the quantification of foods, thus addressing the need to communicate the concept of proportionality within messages. Further discussion is required to determine how to attain this and with which types of foods. For example, as there is no evidence contraindicating an elevated consumption of fruits and vegetables, it was recommended that a minimum intake be established whilst leaving the upper limit open-ended. In other cases, it would be more appropriate to set only upper limits.

5. To reassess the graphic chosen for food-based guidelines. As a supplement to the currently used pyramid, a graphic of a food basket was suggested, which constitutes a concept familiar to many
Mediterranean cultures. The food basket and other variations could be adapted to country-specific consumption trends.

6. To evaluate the magnitude of changes occurring in traditional Mediterranean food patterns. Characteristics of traditional dietary patterns include a variety of factors: frugality, diversity, seasonal variation, proportion and balance between foods, a predominance of vegetable products, use of olive oil, moderate wine consumption and the elaboration of unique dishes, as well as rich and varied culinary expressions and gastronomy. However, current consumption trends (especially in European Mediterranean countries) do not correspond to the traditional concept of Mediterranean dietary patterns, to which globalisation is adding new changes/patterns.

7. To identify the impact of technology and the incorporation of 'non-Mediterranean' foods on consumption patterns. Foods native to the Mediterranean include cereals (wheat, barley, rice, rye), olives and olive oil, grapes and wine, pulses, nuts, fruits and vegetables, and to a lesser extent dairy products (cheese and yoghurt), fish, eggs, lamb, goat, poultry and pork. This has been modified over time with the incorporation of new foods and methods of preparation. Some have enriched and others have worsened the traditional Mediterranean dietary model, whose principles in times past prevailed in the cradle of great civilisations.

8. To distinguish between traditional Mediterranean fare consumed on a day-to-day basis (with reduced proportions of animal products) and that prepared for feast days or celebrations (incorporating sweets and more meat and meat products). Food guides should also reflect these differences.

9. To promote the Mediterranean diet as a way of life, thus establishing its role as a constituent of the cultural heritage of the countries in the region. Apart from being a healthy way of eating, it encompasses history, culture and the art of good living.

10. To establish nutritional criteria for classifying a food as Mediterranean that is not strictly limited to geographic location, which is the basis for the current model derived from 1960s' food data. Historically, Mediterranean dietary patterns reflect an amalgam of food products and influences resulting from the interchange of diverse cultures all along its shores. Mediterranean food patterns of the future should maintain this cross-cultural aspect, being adaptable enough to incorporate foods/products that are healthy but not necessarily considered traditionally Mediterranean, with the exception of those inducing a loss of the essential Mediterranean character and identity. 
This workshop aimed to establish the basis for a diverse variety of actions in the future, among which figure accompanying measures and concerted actions within the European Commission.

\section{The International Task Force on the Mediterranean Diet}

Chairman: Lluis Serra-Majem (Foundation for the Advancement of the Mediterranean Diet, Barcelona Science Park, Community Nutrition Research Group, University of Barcelona and University of Las Palmas de Gran Canaria, Spain).

Members: Javier Aranceta (Community Nutrition Unit, Bilbao City Council, Bilbao, Spain), Victoria Arija (University of Rovira i Virgili, Reus, Spain), JA Amorim Cruz (Nutrition Research Centre, National Institute of Health, Portugal), Maurizio Battino (University of Ancona, Italy), Antonio Bayés de Luna (Hospital de la Santa Creu y Sant Pau de Barcelona, Spain), Luis Bello (University of Las Palmas de Gran Canaria, Spain), Elliot Berry (Hebrew University, Israel), Pilar Cervera (Centre d'Ensenyament Superior de Nutrició i Dietètica, University of Barcelona, Spain), Ramon Clotet (University of Barcelona, Spain), Amleto D'Amicis (Unit of Food Economics and Statistics, INRAN, Italy), Sandro Dernini (EuroMed Forum, Italy), Ismael Díaz Yubero (Ministry of Health and Consumption, Spain), M- Carmen Fuentes (Ministry of Agriculture, Fisheries and Food, Spain), Carlos Alberto González (Catalan Institute of Oncology, Spain), Carlo La Vecchia (University of Milan, Italy), Bill Layden (Porter Novelli, USA), Aicha Lemtouni (Institut Agronomique et Vétérinaire Hassan II, Morocco), José Mataix (University of Granada, Spain), Xavier Medina (Institut Europeen de la Mediterrania, Spain), Marion Nestle (New York University, USA), Joy Ngo de la Cruz (Foundation for the Advancement of the Mediterranean Diet, Barcelona Science Park,
Centre d'Ensenyament Superior de Nutrició i Dietètica, University of Barcelona, Spain), Dorit Nitzan (Department of Nutrition, Ministry of Health, Israel), Gülden Pekcan (Hacettepe University, Department of Nutrition and Dietetics, Turkey), Carmen Pérez Rodrigo (Community Nutrition Unit, Bilbao City Council, Bilbao, Spain), Jordi Salas (University of Rovira i Virgili, Reus, Spain), Llorenç Torrado (Foundation for the Advancement of the Mediterranean Diet, Spain), Antonia Trichopoulou (University of Athens Medical School, Greece), Dimitrios Trichopoulos (Harvard School of Public Health, USA) and M Carmen Vidal Carou (University of Barcelona, Spain).

\section{Acknowledgements}

The authors are grateful to Eva Álvarez, Alicia García Álvarez, Blanca Gonzalvo, Gabriela Nicola, Imma Palma, Lourdes Ribas and Gemma Salvador for their support in the organisation of the Task Force.

\section{References}

1 Helsing E, Trichopoulou A, eds. The Mediterranean diet and food culture - a symposium. European Journal of Clinical Nutrition 1989; 43(Suppl. 2): 1-92.

2 Serra Majem L, Helsing E, ed. Changing patterns of fat intake in Mediterranean countries. European Journal of Clinical Nutrition 1993; 47(Suppl. 1): S1-S100.

3 Nestle M, ed. Mediterranean diets: science and policy implications. American Journal of Clinical Nutrition 1995; 61(Suppl. 6): 1313S-427S.

4 Willett WC, Sacks F, Trichopoulou A, Drescher G, Ferro-Luzzi A, Helsing E, et al. Mediterranean diet pyramid: a cultural model for healthy eating. American Journal of Clinical Nutrition 1995; 61(Suppl. 6): 1402S-6S.

5 Trichopoulou A, Lagiou P. Healthy traditional Mediterranean diet: an expression of culture, history, and lifestyle. Nutrition Reviews 1997; 55: 383-9.

6 Serra Majem L, Ngo de la Cruz J, eds. ¿Qué es la Dieta Mediterránea? Barcelona: Nexus Ediciones, 2002; 1-221. 